\title{
Theranosties
}

Review

2011; 1:395-402

\section{Lasting Controversy on Ranibizumab and Bevacizumab}

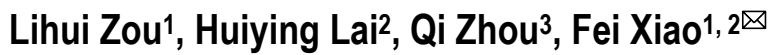

1. The Key Laboratory of Geriatrics, Beijing Hospital \& Beijing Institute of Geriatrics, Ministry of Health, Beijing, 100730, China.

2. The Department of Laboratory Medicine, Beijing Hospital, Ministry of Health, Beijing 100730, China.

3. National Center for Clinical Laboratories, Ministry of Health, Beijing 100730, China.

Corresponding author: Fei Xiao, The Key Laboratory of Geriatrics, Beijing Institute of Geriatrics; The Department of Laboratory Medicine, Beijing Hospital, Beijing 100730, China. E-mail: xiaofei@bjhmoh.cn

(C) Ivyspring International Publisher. This is an open-access article distributed under the terms of the Creative Commons License (http://creativecommons.org/ licenses/by-nc-nd/3.0/). Reproduction is permitted for personal, noncommercial use, provided that the article is in whole, unmodified, and properly cited.

Received: 2011.09.20; Accepted: 2011.11.29; Published: 2011.12.12

\begin{abstract}
Vascular endothelial growth factor (VEGF), an important angiogenic factor that is able to stimulate the proliferation and migration of endothelial cells, is the best-studied hallmark of angiogenesis. Neovascularization is a major cause of age-related macular degeneration (AMD) which is a leading cause of blindness in the elderly population. Specific molecular inhibitors of VEGF have been proved to be useful in the treatment of AMD. Ranibizumab and Bevacizumab are structurally similar to anti-VEGF drugs in the treatment of AMD. Many studies have indicated that Ranibizumab and Bevacizumab are of roughly equal short-term efficacy and safety, Bevacizumab is an attractive alternative to Ranibizumab due to its lower cost. However, only Ranibizumab has received Food and Drug Administration (FDA) approval for the treatment of macular degeneration. More multicenter clinical trials are required to compare the relative efficacy and safety of these two drugs and some progress has been achieved. This review discusses the clinical effectiveness, safety, cost and other practical implications of Ranibizumab and Bevacizumab.
\end{abstract}

Key words: Ranibizumab; Bevacizumab; vascular endothelial growth factor (VEGF); age-related macular degeneration; angiogenesis.

\section{Introduction}

Vascular endothelial growth factor (VEGF) and its receptor (Flt/VEGFR family) system play a critical role not only in physiological but also in most parts of pathological angiogenesis including age-related macular degeneration (AMD). In recent years, several laboratories have dedicated to research on the important members involved in angiogenesis, particularly on VEGF-VEGFRs. VEGF binding to VEGF receptor-2 (VEGFR-2) starts a tyrosine kinase signaling cascade in endothelial cells that stimulates the production of factors to be associated with vascular permeability (eNOS) [1], proliferation/survival (bFGF) [2], migration (ICAMs/VCAMs/MMPs) [3] and fi- nally differentiation into mature blood vessels as shown in Figure 1.

Angiogenesis refers to the physiological process of the growth of new blood vessels. It is a normal and vital process for growth, development, wound healing and reproduction [4]. Angiogenesis is a complex process involving angiogenic factors, endothelial cells and extracellular matrix (ECM) components. The process undergoes three well-characterized phases of development. First, angiogenic growth factors activate receptors that are expressed on the endothelial cells following the biological signal stimulation. Second, the activated endothelial cells start to release 
enzymes known as proteases which degrade the basement membrane to allow endothelial cells to spread from the primary vessel walls. Third, the proliferative endothelial cells together with the surrounding matrix form solid sprouts connecting neighboring vessels.

When the precise balance between growth and inhibitory factors of angiogenesis is disturbed, the excessive or insufficient angiogenesis results in the growth of abnormal blood vessels which is dramatically involved in the development of age-related blindness, cancer, skin diseases, stroke, diabetic ulcers, cardiovascular disease and many other diseases.

Angiogenesis is a major contributor to AMD, the fastest growing form of macular degeneration. There are 20 million new cases of AMD in the United States and millions more worldwide each year. Two types of AMD, "wet" or neovascular and "dry" or atrophic have been well studied. Nowadays, most treatments are available for the "wet" AMD. Several anti-VEGF drugs have been developed, after vascular endothelial growth factor A (VEGFA) was found to be a key agent in neovascularization and vascular leakage in AMD [5].

Currently, Ranibizumab (Lucentis) and Bevacizumab (Avastin) are two major anti-VEGF drugs for the treatment of AMD. Ranibizumab was licensed by FDA in June 2006, while Bevacizumab with similar molecular structure to Ranibizumab has also been proved to be highly effective for AMD. More importantly, the latter is much cheaper, and therefore is economically meaningful for lower-income populations. However, up to now only Bevacizumab has been approved by FDA for the treatment of some malignancies such as colon cancer, but not for the treatment of macular degeneration. As an alternative, many ophthalmologists are using Bevacizumab "off-label" to treat AMD. Controversies remain over whether Ranibizumab or Bevacizumab is superior in treating macular degeneration. Therefore, this review, based on our knowledge of VEGF and its receptors as well as the AMD, tries to compare the clinical advantages and disadvantages of these two drugs.

\section{VEGF and VEGF receptors}

VEGF family includes VEGF-A, VEGF-B, VEGF-C, VEGF-D, VEGF-E and placental growth factor (PlGF), among which the most important member is VEGF-A [6]. The VEGF family has distinct binding affinities for VEGFR-1, -2 , and -3 . Members of VEGF family contain a common structure of eight characteristically spaced cysteine residues in a VEGF homology domain. VEGF-A was first identified in the media conditioned by bovine pituitary follicular cells [7]. It is a $34-$ to $42-\mathrm{kDa}$, dimeric, disulfide-bound glycoprotein that is specifically activated on endothelial cells and plays a key role in various processes such as inducing angiogenesis, accelerating the endothelial cell growth, promoting cell migration, and inhibiting apoptosis and tumor growth.

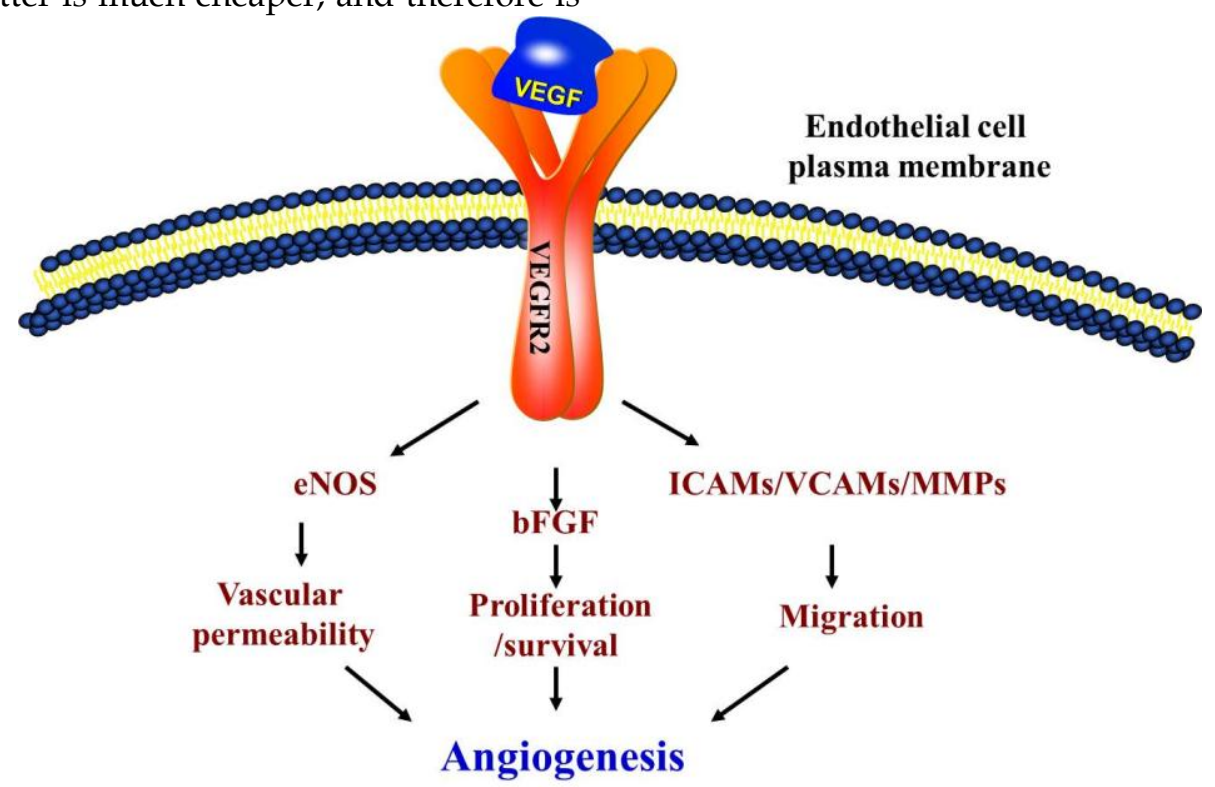

Figure I. Schematic of vascular endothelial growth factor (VEGF) pathway. VEGFR2 is the main signal transducing VEGF receptor for angiogenesis and mitogenesis of endothelial cells. In endothelial cells, the VEGF signal system can adjust the vascular permeability through eNOS. However, the developing vasculature also requires other signaling pathways, including the FGF and the ICAMs/VCAMs/MMPs, and so on. 
At least six VEGF-A isoforms including $\mathrm{VEGF}_{121}, \mathrm{VEGF}_{145}, \mathrm{VEGF}_{165}, \mathrm{VEGF}_{183}, \mathrm{VEGF}_{189}$, and $\mathrm{VEGF}_{206}$ are produced by alternative eight exon splicing of the VEGFA gene [8]. Among these isoforms, $\mathrm{VEGF}_{121}$ and $\mathrm{VEGF}_{165}$ are predominantly expressed in the eye [9]. At least two different mechanisms may control the availability of VEGF-A proteins in endothelial cells: one as freely diffusible proteins, such as VEGF 121 and $\mathrm{VEGF}_{165}$; another one as its longer isoforms, such as $\mathrm{VEGF}_{189}$ and $\mathrm{VEGF}_{206}$, which release into a soluble and bioactive form locally from their bound state by protease cleavage or other means [10].

There are three receptor tyrosine kinases that mediate the angiogenic functions of VEGF family members: VEGFR-1/Flt-1, VEGFR-2/KDR/Flk-1, and VEGFR3/Flt-4. All of them have an extracellular portion including 7 immunoglobulin-like domains, a single transmembrane spanning region, and an intracellular portion containing a split tyrosine-kinase domain [11]. VEGFR-1 and VEGFR-2 are expressed predominantly in vascular endothelial cells and exert important effects on the physiological functions. VEGFR-3 is mainly found in venous endothelium during early embryonic development and then becomes restricted in lymphatic endothelial cells and certain fenestrated blood vascular endothelial cells in the adults [12].

Several lines of evidence have revealed that VEGF has potent pro-angiogenic effects in the retina and choroids; for example, the significantly increased VEGF immunoreactivity is associated with the diabetic retina and choroid [13] and VEGF levels are elevated in the vitreous of patients with subretinal neovascularization [14], which suggests that VEGF may contribute to the increased vascular permeability and angiogenesis. VEGF overexpression may promote the pathological neovascularization by directly stimulating angiogenesis, sustaining endothelial cell survival via inhibiting apoptosis, and/or enhancing vascular permeability by the formation of endothelial fenestrations that predispose to haemorrhage and exudation. Moreover, it up-regulates the expression of matrix metalloproteinases, enzymes that break down extracellular matrix and thus facilitate the invasion of new vessels into the tissues [15].

\section{Age-related macular degeneration (AMD)}

AMD is a common disease that usually affects the older adults ( $>50$ years) and results in a loss of vision in the center of the visual field (the macula). Although children and adolescences can also suffer from an inherited form of macular degeneration often known as "macular dystrophy", this condition is rare.
The retina normally locates in the inner layer of the eye and contains optic nerves. At the back of the retina lies the choroid, which contains the blood supply to all three layers of the eye, including the macula, an oval-shaped highly pigmented yellow spot near the center of the retina; it surrounds the optic disc. At the early stage of AMD, most people have good vision. As the disease develops into the advanced stage, $80-90 \%$ of patients will develop dry or nonexudative AMD, during which waste cellular debris (or "drusen") accumulates between the retina and the choroid, and the retina can become detached. When the drusen gets bigger and denser and the pigmented cell layer under the macula becomes disturbed, the vision begins to degrade.

On the contrary, wet or neovascular, exudative AMD, resulting from abnormal blood vessel growth (choroidal neovascularization, $\mathrm{CNV}$ ) in the choriocapillaris, is more severe, and approximately $90 \%$ of vision loss is due to this type. Patients with wet AMD can suffer from retinal detachment. The new abnormal vessels are fragile, and often extravasate blood components, which occasionally lead to subretinal or vitreous haemorrhage, resulting in sudden visual disturbance. Although the exact mechanisms for the development of CNV are poorly understood, tissue hypoxia and VEGF overexpression may play important roles. For example, hypoxia-inducible factor-1(HIF-1) plays a crucial role in the activation of VEGF genes involved in the angiogenesis processes of CNV [16]. Elevated VEGF levels have been found in laser-induced CNV animal models [17] and in surgically excised CNV membranes [18], AMD patients with active CNV have significantly higher plasma VEGF levels than normal controls [19].

The neovascular AMD can be treated with laser coagulation, or with angiogenesis inhibitor that stops and sometimes reverses the growth of blood vessels. Unfortunately, no highly effective treatment is currently available for wet AMD. However, new drugs known as anti-angiogenic or anti-VEGF agents have been found to be able to reverse the growth of abnormal blood vessels and improve vision after having been injected directly into the vitreous humor of the eye without bringing damage to the retina. Although the current therapies still cannot cure AMD, an increasing number of anti-VEGF agents are under investigation.

\section{Ranibizumab (Lucentis)}

A clinical trial study on VEGF inhibition in ocular neovascularization in late 2004 initiated a new era of AMD treatment, this trial demonstrated that 
VEGF-targeted treatment could affect the formation of neovascular vessels in patients with AMD. The research team firstly confirmed that pegaptanib (Macugen) appeared to be an effective therapy for neovascular AMD [20]. Ranibizumab also is a VEGF-targeted agent. Today, it has been widely used as the first-line treatment for neovascular AMD. Ranibizumab is a recombinantly produced, humanized monoclonal antibody fragment (Fab) designed to bind and inhibit all biologically active isoforms of human VEGF, in contrast to pegaptanib that binds to a single isoform [21]. It has only one antigen-binding domain and is administered via intravitreal injection [22]. Since the mature product is a small molecule and can deeply penetrate into the tissues, it is especially effective for $\mathrm{CNV}$ beneath retinal pigment epithelial cells.

More recently, the Minimally Classic/Occult Trial of the Anti-VEGF Antibody to Ranibizumab in the treatment of neovascular age-related macular degeneration (MARINA) (in which 716 patients were randomly treated with intravitreal injections of 0.3 or $0.5 \mathrm{mg}$ Ranibizumab or sham injections) demonstrated that intravitreal administration of Ranibizumab for 24 months prevented vision loss and improved mean visual acuity. More specifically, the mean increases in visual acuity were 6.5 letters in the $0.3-\mathrm{mg}$ group and 7.2 letters in the $0.5-\mathrm{mg}$ group, as compared with a decrease of 10.4 letters in the sham-injection group. In addition, the rates of severe adverse events were lower in patients with minimally classic or occult (without classic lesions) choroidal neovascularization secondary to AMD. The benefit in visual acuity was maintained for 2 years [23]. Thanks to the amazing results of this trial, Ranibizumab was licensed by the US FDA in 2006 for the treatment of advanced or wet AMD. In another study on the role of Ranibizumab for neovascular AMD, the anti-VEGF antibody for treatment of predominant classic choroidal neovascularization in age-related macular degeneration (ANCHOR) group also found that Ranibizumab improved visual acuity at one year on average, with low rates of severe ocular adverse events. In the ANCHOR study, the mean visual acuity increased by 8.5 letters in the $0.3-\mathrm{mg}$ group and 11.3 letters in the $0.5-\mathrm{mg}$ group, as compared with a decrease of 9.5 letters in the control group [24].

The most common adverse effects of intravitreal Ranibizumab include conjunctival haemorrhage, eye pain and vitreous floaters. In the MARINA study, the incidences of endophthalmitis and severe uveitis after intravitreal administration of Ranibizumab were $1.0 \%$ (5 of 477 patients) and 1.3\% (6 of 477 patients), respectively. However, the incidences of systemic com- plications such as hypertension and arterial thrombolic events showed no significant difference between the Ranibizumab group and control group [23]. In the ANCHOR study, the incidences of endophthalmitis and severe uveitis were $0.7 \%$ ( 2 of 277 patients) and $0.4 \%$ respectively in the Ranibizumab group, with increased immunoreactivity to Ranibizumab in 0.5-mg Ranibizumab group. Transient change in the intraocular pressure after injection was common in the Ranibizumab group. Again, there was no significant difference in the rates of arterial thrombolic events between the treatment groups [24]. Machalinska et al also indicated that intravitreal Ranibizumab did not induce significant systemic effects or vascular impairment [25].

\section{Bevacizumab as good as Ranibizumab}

Like Ranibizumab, Bevacizumab is another monoclonal antibody that binds and inhibits all isoforms of VEGF with a lower affinity. As a larger molecule, Bevacizumab has a longer half-life about 17-21 days [26] and has two antigen-binding domains. Intravenous Bevacizumab was approved by the FDA for the treatment of metastatic colorectal cancer in February 2004. At that time, Bevacizumab was not regarded as a useful agent for the treatment of AMD because this full-length antibody was not believed to be able to penetrate the retina [27], according to the observation that molecules larger than $77 \mathrm{kDa}$ could not freely diffuse across fixed human retina [28]. However, in 2005, Rosenfeld et al. reported that visual acuity and macular appearance were improved under optical coherence tomography (OCT) after single eye treatment of neovascular AMD with intravitreal Bevacizumab. Since then, off-label use of intravitreal Bevacizumab has become an alternative for patients who were not suitable for or refractory to other approved therapies. Meanwhile, animal studies also have found that this particular full-length antibody could penetrate all retinal layers [26].

Several clinical trials have demonstrated that Bevacizumab was beneficial for the treatment of AMD without severe adverse effects [26, 29, 30]. Although no formal dose-ranging or dose-frequency study has been performed, the most commonly used dose in the published literatures is $1.25 \mathrm{mg}$ [31]. In the past, safety was a major concern on the off-label use of Bevacizumab for macular degeneration, especially when US FDA warned in January 2005 that Bevacizumab, when used to treat colon cancer and other malignancies, significantly increased the risk of stroke, heart attack, and other related adverse events [32, 33]. However, according to the results of the International Intravitreal Bevacizumab Safety Survey, although there were 
some procedure-related adverse events and systemic adverse events after the intravitreal injection of Bevacizumab, these adverse events occurred in less than $0.21 \%$ of the patients after 7,113 injections in total [34]. Furthermore, Shima et al. reported that the incidence of complications (total 707 patients) within two months after intravitreal injection was less than $0.42 \%$ [35]. Therefore, it is easy to conclude that the short-term safety of intravitreal Bevacizumab is satisfactory, although the long-term safety remains unknown.

\section{The difference between Ranibizumab and Bevacizumab}

Anti-VEGF therapies have been widely accepted to treat AMD. The epitope of Ranibizumab and Bevacizumab locate in the receptor-binding region of VEGF, both antibodies target VEGF in a similar way. What is the difference? First, Bevacizumab (149 kDa) and Ranibizumab (48.39 kDa) have different molecular weights, mainly because Ranibizumab does not contain an Fc part; second, Bevacizumab is produced in a eukaryotic cell line ( $\mathrm{CHO}$ cells) and is $\mathrm{N}$-glycosylated in its Fc region, while Ranibizumab is produced in prokaryotic E. coli, and therefore it does not carry any glycosylation sites [36]. Both Bevacizumab and Ranibizumab neutralize VEGF and appear to have lasting effects even after they disappear in the culture medium [37].

Controversies remain in the past years over whether Ranibizumab or Bevacizumab is superior in treating macular degeneration. Several recent animal experiments did not offer enough evidences for detecting the important differences in the efficacy and safety of intravitreal Bevacizumab and Ranibizumab in the treatment of neovascular age-related macular degeneration (nAMD) [38, 39]. Fong et al. concluded that both Ranibizumab and Bevacizumab groups showed similar improvement and stability of vision over time [40]. Serum concentrations of both drugs following intravitreal administration were extremely low [41, 42]. However, Ranibizumab shows greater efficiency when it is highly diluted.

In terms of ocular and systemic safety, endophthalmitis, conjunctival haemorrhage, and thromboembolic events such as angina, heart attacks and strokes remain the most common problems. Rich experiences in the usage of both Ranibizumab and Bevacizumab have well demonstrated that the severe adverse events showed no significant differences between the intravitreal Ranibizumab and intravitreal Bevacizumab groups $[43,44]$. Choi et al. found the sustained elevated intraocular pressures after intravitreal injection of Bevacizumab, Ranibizumab, and pegaptanib. The elevated intraocular pressure also showed no association with the type, frequency, and dose of the anti-VEGF agents [45].

Since it has been widely recognized that Bevacizumab is equally effective and safe as Ranibizumab, it is controversial for the curative effects and safeties to switch one treatment for another when it does not work well. Patients with CNV who developed tachyphylaxis after the use of Ranibizumab or Bevacizumab may respond well to another anti-VEGF drug. In a clinical study, $81 \%$ cases demonstrated some responses after switching therapies [46]. However, Karagiannis et al. indicated that the change of treatment from Bevacizumab to a less than half-sized molecule (e.g. Ranibizumab) with less half-life in the vitreous contributed to a transient "instability" in the eye that might have triggered the large subretinal hemorrhage [47].

Furthermore, it is still a question whether Bevacizumab really shares identical efficacy and safety with Ranibizumab, at least for some authors. They argue that anibizumab, as a US FDA-approved agent for AMD, has been well studied in many randomized clinical trials with more long-term findings when compared with Bevacizumab. Ranibizumab represents greater activity in vitro; as a smaller molecule, it is easier to penetrate the eye's retina and inhibit abnormal blood vessel growth. Therefore, they believe Ranibizumab is more effective and safer than Bevacizumab. For example, in Chang et al.'s retrospective comparative study, the authors concluded that the short-term effectiveness of Ranibizumab treatment, as measured by incremental improvement in OCT parameters, was significantly better than Bevacizumab treatment [48].

Similarly, a Japanese randomized trial reported that 5 patients $(14.3 \%)$ developed severe intraocular inflammation after an intravitreal injection of the same dose of Bevacizumab [49]. Hoffmann-La Roche reported 32 cases of endophthalmitis after the off-label intravitreal use of Bevacizumab in Canada. Recently, acute postoperative endophthalmitis developed in 55 of 116 patients after intravitreal injection of Bevacizumab in Shanghai, China [50]. Therefore, the safety of Bevacizumab should be further studied.

Furthermore, many clinicians have an impression that Bevacizumab seems to be longer-acting than Ranibizumab, perhaps because Bevacizumab impedes clearance from the eye based on its larger size. It has been concluded that the degeneration of photoreceptors cells, primarily in the macula, may account for the loss of central vision of patients suffering from a variety of eye diseases including macular degeneration, especially when Bevacizumab treatment is performed 
at higher doses and/or for a longer period of time [51]. Consequently, instead of using the existing treatment schemes, many clinicians prefer to choose a regimen using less frequent dosing of Bevacizumab than Ranibizumab.

Does this mean that Ranibizumab is better than Bevacizumab in treating AMD? Not necessarily. It has been indicated that Bevacizumab accumulated within retinal pigment epithelial (RPE) cells, possibly inducing long-term effects; on the contrary, Ranibizumab did not accumulate [52]. Therefore, there may be substantial differences in the pharmacokinetics of these two macular degeneration drugs.

Moreover, Ranibizumab is expensive which costs to exceed \$9 billion per year in U.S. and one Ranibizumab injection is expected to cost $\$ 1950$. Fortunately, the cost for each Bevacizumab injection is only about $\$ 50$. The cost-effectiveness should always be considered when making clinical decisions. Considering its low cost and comparable efficacy/safety, many doctors and patients would agree that Bevacizumab is a better choice in treating AMD. Table 1 summarizes the sameness and difference between Ranibizumab and Bevacizumab. Actually, more multicenter clinical trials are comparing the effectiveness and safety of these two drugs.

\section{Possible choices for other diseases besides AMD}

Besides AMD, VEGF is also a main factor of other eye diseases including diabetic macular edema (DME), central retinal vein occlusion (CRVO), retinal vascular thrombosis and $\mathrm{CNV}$ caused by other dis- orders such as high myopia. Since VEGF-targeted drugs work well for wet AMD, they may also be effective and safe for these diseases mentioned above. For example, intraocular injections of $0.5-\mathrm{mg}$ Ranibizumab significantly reduced foveal thickness and improved visual acuity in patients with chronic DME [53]. However, a randomized, controlled and double-masked trial is urgently needed to clarify whether intraocular injection of Ranibizumab provides long-term benefit to patients with DME. Actually, Ferrara et al. had evaluated the effectiveness of intravitreal Bevacizumab for CRVO. In their study, patients with CRVO of fewer than three-month duration received intravitreal Bevacizumab as the primary treatment. The patients experienced a dramatic improvement in the visual acuity and clinical fundus appearance without collateral vessel formation [54]. This finding also implies that early initiation of anti-VEGF treatment should be further studied in a larger trial for CRVO.

\section{Conclusions}

The past decade has witnessed the promising potential effect of Ranibizumab and Bevacizumab in treating AMD due to their potent anti-VEGF properties. With similar structure, Ranibizumab and Bevacizumab have their certain dose regimen, effectiveness and safety. However, it still remains controversial whether Ranibizumab or Bevacizumab has the better cost-effectiveness for AMD treatment. More rigorously designed multicenter clinical trials are urgently needed to evaluate the long-term effectiveness and safety of these two drugs.

Table I. The sameness and difference between Ranibizumab and Bevacizumab.

\begin{tabular}{lll}
\hline & Ranibizumab & Bevacizumab \\
\hline The sameness & VEGF & VEGF \\
Target & Monoclonal antibody & Monoclonal antibody \\
Product & Receptor-binding region & Receptor-binding region \\
Epitope & & \\
The difference & $48.39 \mathrm{kDa}$ & $149 \mathrm{kDa}$ \\
Molecular weight & 3 days & $17-21$ days \\
Half-life & No & Yes \\
Glycosylate & E. coli & CHO cells \\
Expression system & Strong & Strong \\
Effectiveness & Uncertain & Uncertain \\
Safety & $\$ 1950$ per dose & $\$ 50$ per dose \\
Cost & For AMD & For cancer \\
FDA-approved & &
\end{tabular}




\section{Conflict of Interest}

The authors have declared that no conflict of interest exists.

\section{References}

1. Fukumura D, Gohongi T, Kadambi A, Izumi Y, Ang J, Yun CO, et al. Predominant role of endothelial nitric oxide synthase in vascular endothelial growth factor-induced angiogenesis and vascular permeability. Proc Natl Acad Sci U S A. 2001; 98 : 2604-9.

2. Kigel B, Rabinowicz N, Varshavsky A, Kessler O, Neufeld G. Plexin-A4 promotes tumor progression and tumor angiogenesis by enhancement of VEGF and bFGF signaling. Blood. 2011; 118: 4285-96.

3. Hollborn M, Stathopoulos C, Steffen A, Wiedemann P, Kohen L, Bringmann A. Positive feedback regulation between MMP-9 and VEGF in human RPE cells. Invest Ophthalmol Vis Sci. 2007; 48: 4360-7.

4. Maragoudakis ME. Angiogenesis in health and disease. Gen Pharmacol. 2000; 35: 225-6.

5. Klagsbrun M, D'Amore PA. Regulators of angiogenesis. Annu Rev Physiol. 1991; 53: 217-39.

6. Hoeben A, Landuyt B, Highley MS, Wildiers H, Van Oosterom AT, De Bruijn EA. Vascular endothelial growth factor and angiogenesis. Pharmacol Rev. 2004; 56: 549-80.

7. Ferrara N, Henzel WJ. Pituitary follicular cells secrete a novel heparin-binding growth factor specific for vascular endothelial cells. Biochem Biophys Res Commun. 1989; 161: 851-8.

8. Levy AP, Levy NS, Loscalzo J, Calderone A, Takahashi N, Yeo $\mathrm{KT}$, et al. Regulation of vascular endothelial growth factor in cardiac myocytes. Circ Res. 1995; 76: 758-66.

9. Sassa Y, Hata Y. Antiangiogenic drugs in the management of ocular diseases: Focus on antivascular endothelial growth factor. Clin Ophthalmol. 2010; 4: 275-83.

10. Park JE, Keller GA, Ferrara N. The vascular endothelial growth factor (VEGF) isoforms: differential deposition into the subepithelial extracellular matrix and bioactivity of extracellular matrix-bound VEGF. Mol Biol Cell. 1993; 4: 1317-26.

11. Ortega N, Hutchings $H$, Plouet J. Signal relays in the VEGF system. Front Biosci. 1999; 4: D141-52.

12. Kaipainen A, Korhonen J, Mustonen T, Van Hinsbergh V, Fang $\mathrm{GH}$, Dumont D, et al. Expression of the fms-like tyrosine kinase 4 gene becomes restricted to lymphatic endothelium during development. Proc Natl Acad Sci U S A. 1995; 92: 3566-70.

13. Lutty GA, McLeod DS, Merges C, Diggs A, Plouet J. Localization of vascular endothelial growth factor in human retina and choroid. Arch Ophthalmol. 1996; 114: 971-7.

14. Wells JA, Murthy R, Chibber R, Nunn A, Molinatti PA, Kohner $\mathrm{EM}$, et al. Levels of vascular endothelial growth factor are elevated in the vitreous of patients with subretinal neovascularisation. Br J Ophthalmol. 1996; 80: 363-6.

15. Ng EWM, Adamis AP. Targeting angiogenesis, the underlying disorder in neovascular age-related macular degeneration. Can J Ophthalmol. 2005; 40: 352-68.

16. Arjamaa O, Nikinmaa M. Oxygen-dependent diseases in the retina: role of hypoxia-inducible factors. Exp Eye Res. 2006; 83: 473-83..

17. Yi X, Ogata N, Komada M, Yamamoto C, Takahashi K, Omori $\mathrm{K}$, et al. Vascular endothelial growth factor expression in choroidal neovascularization in rats. Graefes Arch Clin Exp Ophthalmol. 1997; 235: 313-9.

18. Tolentino MJ, Mcleod DS, Taomoto M, Otsuji T, Adamis AP, Lutty GA. Pathologic features of vascular endothelial growth factor-induced retinopathy in the nonhuman primate. Am J Ophthalmol. 2002; 133: 373-85.

19. Tsai DC, Charng MJ, Lee FL, Hsu WM, Chen SJ. Different plasma levels of vascular endothelial growth factor and nitric oxide between patients with choroidal and retinal neovascularization. Ophthalmologica. 2000; 220: 246-51.

20. Gragoudas ES, Adamis AP, Cunningham Jr ET, Feinsod M, Guyer DR. Pegaptanib for neovascular age-related macular degeneration. N Engl J Med. 2004; 351: 2805-16.

21. Zarbin M, Szirth B. Current treatment of age-related macular degeneration. Optom Vis Sci. 2007; 84: 559-72.

22. Gaudreault J, Fei D, Rusit J, Suboc P, Shiu V. Preclinical pharmacokinetics of Ranibizumab (rhuFabV2) after a single intravitreal administration. Invest Ophthalmol Vis Sci. 2005; 46: 726-33.

23. Rosenfeld PJ, Brown DM, Heier JS, Boyer DS, Kaiser PK, Chung CY, et al. Ranibizumab for neovascular age-related macular degeneration. N Engl J Med. 2006; 355: 1419-31.

24. Brown DM, Kaiser PK, Michels M, Soubrane G, Heier JS, Kim RY, et al. Ranibizumab versus verteporfin for neovascular age-related macular degeneration. N Engl J Med. 2006; 355: 1432-44.

25. Machalinska A, Paczkowska E, Pabin T, Safranow K, Karczewicz D, Machalinski B. Influence of ranibizumab on vascular endothelial growth factor plasma level and endothelial progenitor cell mobilization in age-related macular degeneration patients: safety of intravitreal treatment for vascular homeostasis. J Ocul Pharmacol Ther. 2011; 27: 471-5.

26. Avery RL, Pieramici DJ, Rabena MD, Castellarin AA, Nasir MA, Giust MJ. Intravitreal bevacizumab (Avastin) for neovascular age-related macular degeneration. Ophthalmology. 2006; 113: 363-72.

27. Mordenti J, Cuthbertson RA, Ferrara N, Thomsen K, Berleau L, Licko V, et al. Comparisons of the intraocular tissue distribution, pharmacokinetics, and safety of 125I-labeled full-length and Fab antibodies in rhesus monkeys following intravitreal administration. Toxicol Pathol. 1999; 27: 536-44.

28. Jackson TL, Antcliff RJ, Hillenkamp J, Marshall J. Human retinal molecular weight exclusion limit and estimate of species variation. Invest Ophthalmol Vis Sci. 2003; 44: 2141-6.

29. Aisenbrey S, Ziemssen F, V lker M, Gelisken F, Szurman P, Jaissle $G$, et al. Intravitreal bevacizumab (Avastin) for occult choroidal neovascularization in age-related macular degeneration. Graefe's Archive for Clinical and Experimental Ophthalmology. 2006; 245: 941-8.

30. Fong K, Kirkpatrick N, Mohamed Q, Johnston RL. Intravitreal bevacizumab (Avastin) for neovascular age-related macular degeneration using a variable frequency regimen in eyes with no previous treatment. Clinical \& Experimental Ophthalmology. 2008; 36: 748-55.

31. Schouten JS, La Heij EC, Webers CA, Lundqvist IJ, Hendrikse F. A systematic review on the effect of bevacizumab in exudative age-related macular degeneration. Graefes Arch Clin Exp Ophthalmol. 2009; 247: 1-11.

32. Fernando NH, Hurwitz HI. Targeted therapy of colorectal cancer: clinical experience with bevacizumab. Oncologist. 2004; 9 Suppl 1: 11-8.

33. Ferrara N, Hillan KJ, Novotny W. Bevacizumab (Avastin), a humanized anti-VEGF monoclonal antibody for cancer therapy. Biochem Biophys Res Commun. 2005; 333: 328-35.

34. Fung AE, Rosenfeld PJ, Reichel E. The International Intravitreal Bevacizumab Safety Survey: using the internet to assess drug safety worldwide. Br J Ophthalmol. 2006; 90: 1344-9.

35. Shima C, Sakaguchi H, Gomi F, Kamei M, Ikuno Y, Oshima Y, et al. Complications in patients after intravitreal injection of bevacizumab. Acta Ophthalmol. 2008; 86: 372-6. 
36. Magdelaine-Beuzelin C, Pinault C, Paintaud G, Watier H. Therapeutic antibodies in ophthalmology: old is new again. MAbs. 2010; 2: 176-80.

37. Klettner A, Roider J. Comparison of bevacizumab, ranibizumab, and pegaptanib in vitro: efficiency and possible additional pathways. Invest Ophthalmol Vis Sci. 2008; 49: 4523-7.

38. Abouammoh M, Sharma S. Ranibizumab versus bevacizumab for the treatment of neovascular age-related macular degeneration. Curr Opin Ophthalmol. 2011; 22: 152-8.

39. Raftery J, Clegg A, Jones J, Tan SC, Lotery A. Ranibizumab (Lucentis) versus bevacizumab (Avastin): modelling cost effectiveness. Br J Ophthalmol. 2007; 91: 1244-6.

40. Fong DS, Custis P, Howes J, Hsu JW. Intravitreal bevacizumab and ranibizumab for age-related macular degeneration a multicenter, retrospective study. Ophthalmology. 2010; 117: 298-302.

41. Blick SK, Keating GM, Wagstaff AJ. Ranibizumab. Drugs. 2007; 67: 1199-206.

42. Stewart MW. Predicted biologic activity of intravitreal bevacizumab. Retina. 2007; 27: 1196-200.

43. van der Reis MI, La Heij EC, De Jong-Hesse Y, Ringens PJ, Hendrikse F, Schouten JS. A systematic review of the adverse events of intravitreal anti-vascular endothelial growth factor injections. Retina. 2011; 31: 1449-69.

44. Biswas P, Sengupta S, Choudhary R, Home S, Paul A, Sinha S. Comparative role of intravitreal ranibizumab versus bevacizumab in choroidal neovascular membrane in age-related macular degeneration. Indian J Ophthalmol. 2011; 59: 191-6

45. Choi DY, Ortube MC, McCannel CA, Sarraf D, Hubschman JP, McCannel TA, et al. Sustained elevated intraocular pressures after intravitreal injection of bevacizumab, ranibizumab, and pegaptanib. Retina. 2011; 31: 1028-35.

46. Gasperini JL, Fawzi AA, Khondkaryan A, Lam L, Chong LP, Eliott D, et al. Bevacizumab and ranibizumab tachyphylaxis in the treatment of choroidal neovascularisation. Br J Ophthalmol. 2011, [Epub ahead of print].

47. Karagiannis DA, Ladas ID, Parikakis E, Georgalas I, Kotsolis A, Amariotakis G, et al. Changing from bevacizumab to ranibizumab in age-related macular degeneration. Is it safe? Clin Interv Aging. 2009; 4: 457-61.

48. Chang TS, Kokame G, Casey R, Prenner J, Feiner L, Anderson N. Short-term effectiveness of intravitreal bevacizumab versus ranibizumab injections for patients with neovascular age-related macular degeneration. Retina. 2009; 29: 1235-41.

49. Sato T, Emi K, Ikeda T, Bando H, Sato S, Morita S, et al. Severe intraocular inflammation after intravitreal injection of bevacizumab. Ophthalmology. 2010; 117: 512-6.

50. Cheng JW, Wei RL. Ranibizumab for age-related macular degeneration. N Engl J Med. 2011; 364: 582.

51. Avci B, Avci R, Inan UU, Kaderli B. Comparative evaluation of apoptotic activity in photoreceptor cells after intravitreal injection of bevacizumab and pegaptanib sodium in rabbits. Invest Ophthalmol Vis Sci. 2009; 50: 3438-46.

52. Klettner AK, Kruse ML, Meyer T, Wesch D, Kabelitz D, Roider J. Different properties of VEGF-antagonists: Bevacizumab but not Ranibizumab accumulates in RPE cells. Graefes Arch Clin Exp Ophthalmol. 2009; 247: 1601-8.

53. Nguyen QD, Tatlipinar S, Shah SM, Haller JA, Quinlan E, Sung J, et al. Vascular endothelial growth factor is a critical stimulus for diabetic macular edema. Am J Ophthalmol. 2006; 142: 961-9.

54. Ferrara DC, Koizumi H, Spaide RF. Early bevacizumab treatment of central retinal vein occlusion. Am J Ophthalmol. 2007; 144: 864-71. 